\title{
Correspondence
}

\section{Author's Reply to: Valvular Calcification and Atherosclerosis}

\author{
Yoshihiro Takeda and Masaaki Hoshiga
}

First Department of Internal Medicine, Osaka Medical College, Takatsuki, Japan

We thank Prof. Yetkin and colleagues for their very insightful comments to our article ${ }^{1)}$. They particularly focus on the absence of association between significant coronary stenosis and valvular calcification. They suggest that this finding might be due to the different mechanism of calcified valve formation from "atherosclerosis in the vasculature". We concur with them as calcified valvular process shares some similarities with atherosclerotic process and it has also some differences. In addition to numerous reports including Prof. Yetkin's review ${ }^{2)}$, we recognized that the aortic valve in hypercholeterolemic rabbits showed similar atherosclerotic findings like endothelial disruption and differences like myofibroblast-osteoblast transition ${ }^{3)}$.

We would like to point out an important finding in our study: the prevalence of valvular calcification was low $(24.5 \%$ in aortic valve and $15.4 \%$ in mitral valve) as compared with that of vascular calcification (68.8, 66.4, and 54.5\% in coronary artery, thoracic descending aorta, and thoracic ascending aorta, respectively) in Table $\mathbf{1}$. One possible explanation of these differences is due to different mechanisms of atherosclerotic and calcified processes between in vasculature and in valve. The other one is due to different susceptibility to atherosclerotic changes: valves might be resistant to atherosclerosis like brachial artery ${ }^{4)}$.

We agree with Prof. Yetkin and colleagues that further study is required to elucidate the association of valvular calcification with atherosclerosis. Multi-slice computed tomography enables us a noninvasive evaluation of both coronary stenosis and valvular calcification, so further study with this modality would clarify this important question.

\section{References}

1) Takeda Y, Hoshiga M, Tatsugami F, Morinaga I, Takehara
K, Hotchi J, Yuki T, Ishihara T, Hanafusa T: Clinical significance of calcification in ascending aorta as a marker for the requirement of coronary revascularization. J Atheroscler Thromb, 2009; 16: 346-354

2) Yetkin E, Waltenberger J: Molecular and cellular mechanisms of aortic stenosis. Int J Cardiol, 2009; 135: 4-13

3) Arishiro K, Hoshiga M, Negoro N, Jin D, Takai $S$, Miyazaki M, Ishihara T, Hanafusa T: Angiotensin receptor-1 blocker inhibits atherosclerotic changes and endothelial disruption of the aortic valve in hypercholesterolemic rabbits. J Am Coll Cardiol, 2007; 49: 1482-1489

4) Halperin JL, Creager MK: Arterial obstructive diseases of the extremities. In: Vascular Medicine, ed by Loscalzo J, Creager MK, Dzau VJ: pp835-865, Little, Brown \& Co, Boston, Massachusetts, USA, 1992

Address for correspondence: Yoshihiro Takeda,

E-mail: in1231@poh.osaka-med.ac.jp 